\title{
Grain boundary and curvature enhanced lithium adsorption on carbon
}

\author{
Zhenqian Pang a, Xinghua Shi ${ }^{\mathrm{b}}$, Yujie Wei ${ }^{\mathrm{a}}{ }^{*}$, Daining Fang ${ }^{\mathrm{c}, * *}$ \\ a LNM, Institute of Mechanics, Chinese Academy of Sciences, Beijing 100190, China \\ b CAS Key Laboratory for Nanosystem and Hierarchy Fabrication, CAS Center for Excellence in Nanoscience, National Center for Nanoscience and \\ Technology, Chinese Academy of Sciences, Beijing 100190, China \\ ${ }^{\mathrm{c}}$ Institute of Advanced Structure Technology, Beijing Institute of Technology, Beijing 100081, China
}

\section{A R T I C L E I N F O}

\section{Article history:}

Received 19 February 2016

Received in revised form

20 May 2016

Accepted 13 June 2016

Available online 15 June 2016

\begin{abstract}
A B S T R A C T
While the speculation that graphene may owe double or even higher capacity of lithium adsorption than graphite does remains speculative, there is growing evidence that defects and edges may promote lithium adsorption on graphene and other nanostructured carbon. Here we report a first-principles study on how grain boundary defects in graphene may influence the adsorption of lithium. The adsorption energy for Li atoms trapping in 5-, 7-, and 8-rings is much lower than the counter-part of Li atoms and pristine graphene. Such defective graphene could adsorb more Li atoms, and may reach the speculated ratio of 1:1 for C-Li adsorption. In a contrast study of lithium on fullerenes of different size, we find that the adsorption energy decreases with increasing size of fullerenes, but does not approach the energy when $\mathrm{Li}$ atoms adsorb on flat graphene. The energy in carbon nanotubes, however, converges to the adsorption energy between $\mathrm{Li}$ atoms and flat graphene if the radius of carbon nanotubes is sufficiently large. It hence indicates that while curvature plays a role in the enhanced adsorption in fullerenes, the twelve 5 rings in a fullerene ball is the primary factor accounting for the enhanced lithium adsorption.

(c) 2016 Elsevier Ltd. All rights reserved.
\end{abstract}

\section{Introduction}

As the lithium ion batteries widely utilized in various fields as a type of clean energy, the enhancement of lithium storage in electrode materials becomes a key issue in the design and synthesis of battery with high capacity [1]. It is known that the lithium storage capacity is $372 \mathrm{~mA} \mathrm{~h} / \mathrm{g}$ for graphite [2] and $784 \mathrm{~mA} \mathrm{~h} / \mathrm{g}$ for graphene due to the capture of lithium ions on both surfaces [3]. Carbon nanotubes (CNTs) also owe large specific surface area and are proposed for suitable lithium storage materials as well [4-6]. It was shown that the maximum capacity of CNTs can be as high as $1000 \mathrm{~mA} \mathrm{~h} / \mathrm{g}$ (in the form of $\mathrm{Li}_{2.7} \mathrm{C}_{6}$ ) by chemical etching [7] or ballmilling [8], which facilitates Li ions to diffuse freely inside the CNTs. To further improve the three key characteristics of lithium ion battery performance - capacity, voltage and energy density - other routines were also explored to tune lithium adsorption in carbon. The essence is to manipulate the chemistry, microstructure and shape of an electrode, hence changes the electronic properties of

\footnotetext{
* Corresponding author.

** Corresponding author.

E-mail addresses: yujie_wei@Inm.imech.ac.cn (Y. Wei), fangdn@bit.edu.cn (D. Fang).
}

Li-C interaction [9], to improve binding between lithium ions and the electrode material. Typical routines involve the following three strategies.

(1) Chemical engineering by doping: Recently, Koh et al. investigated $\mathrm{Li}$ atoms embedded in the graphene-fullerene hybrid system [10] and fullerene-CNTs hybrid system [11-13] by first-principle calculations. Heterogeneous structure composed of ultrathin MoS2/nitrogen-doped graphene nanosheets also exhibits highly reversible lithium storage [14]. Alternatively, Wang et al. found that doping boron on graphene can be an effective method to enhance the lithium storage capacity, in which every boron atom can absorb $6 \mathrm{Li}$ ions [15]. Wu et al. followed this line to investigate $\mathrm{Li}$ adsorption on nitrogen doped and boron doped graphenes [16], while Zhou et al. study the Li adsorption on nitrogen doped and boron doped CNTs [17]. Liu et al. also explored the feasibility of lithium storage on graphene and Its derivatives and they reported that certain structural defects in graphene can bind Li stably, yet a more efficacious approach is through substitution doping with boron (B) [18]. Substitutional doping with nitrogen in graphene is revealed to influence the electronic and adsorption properties of graphene 
significantly as well [19]. A very recent work demonstrated that hydrogen could enhance the capacity of graphene anode materials for lithium-ion batteries [20].

(2) Defect engineering: By functionalizing graphene sheets with hierarchical arrangement of pore structures and hence a high number of reactive sites for electrochemical reactions, Xiao et al. realized an exceptionally high capacity electrode-15,000 mA h/g [21]. Both the work by Zhou et al. [22] and Fan et al. [23] Investigated the effect of point defects on the Li adsorption on graphene and reported that the presence of point defects can significantly increase the adsorption of Li on graphene. Meanwhile, Kazume and Nishidate investigated Li adsorption and diffusion on defective CNTs [24]. Recently, Datta et al. [25] further explored the adsorption of Li on graphene with divacancy and Stone-Wales defects. Their results confirmed enhanced Li adsorption on defective graphene because of the increased charge transfer between adatom and underlying defective sheet. At the microscopic level, Mukherjee et al. [26] showed that photoflash and laser-reduced free-standing graphene paper could be high-rate capable anodes for lithium-ion batteries as photothermal reduction of graphene oxide yields an expanded structure with micrometer-scale pores, cracks, and intersheet voids. Their recent experiments illustrated that defect may induce plating of lithium metal within porous graphene networks [27]. Experiments by Lee et al. [28] demonstrated that by controlling defect concentration in graphene sheets, we may attain a high charge capacity of $2310 \mathrm{~mA} \mathrm{~h} / \mathrm{g}$ in lithium-ion batteries, as a resultant of additional lithium storage sites such as defects and edges.

(3) Morphology manipulation. In addition to chemical engineering and microstructure control, morphology is another factor to influence binding between lithium ions and the electrode material $[18,29]$.

Despite the substantial progress on tuning the chemistry, microstructure and shape of an electrode for better binding between lithium ions and the electrode material, how the grainboundary (GB) defects and curvature of carbon structures influencing the Li adsorption remains elusive. For example, 5-7 rings are the most commonly seen grain boundary defects in polycrystalline graphene for engineering practice but their influencing on Li adsorption on graphene is missing. In the morphology side, how curvature affects Li-C binding remains unknown. In this paper, we make a thorough comparison among the binding capability of $\mathrm{Li}$ atoms with different GB defects in graphene. In addition, by examining the evolution of binding energy of $\mathrm{Li}-\mathrm{C}$ in fullerene and CNTs with different size, we demonstrate clearly how the curvature affects the lithium adsorption in those nanostructured carbon. Our results may provide theoretical basis for defect engineering and morphology manipulation to realize high-capacity of graphene anode materials for lithium ion batteries.

\section{Methods}

Density functional theory (DFT) implemented in the Vienna ab initio simulation package (VASP) $[30,31]$ is used in all our calculations. The parameterization by Perdew-Burke-Ernzerhof (PBE) [32] is used for exchange and correlation interaction in the generalized gradient approximation (GGA). We had tested the validity of PBE calculations by examining its predictability for the lattice constant of graphene. The result from PBE calculation is in good agreement with the experiments [33]. A $3 \times 3$ rhombus supercell is used to investigate the interaction of $\mathrm{Li}$ atoms with pristine graphene. The $z$-direction lattice constant is fixed at $20 \AA$ to avoid the layers interaction. The Brillouin zone is sampled by a $15 \times 15 \times 1$ Monkhorst and Pack [34] grid. For (33)-(66) and (77)-(99) CNTs, the $x$ and $y$-direction lattice constants are $20 \times 20 \AA$ and $25 \times 25 \AA$, respectively. The Brillouin zone is sampled by a $1 \times 1 \times 12$ Monkhorst and Pack grid. The xyz-direction lattice constant of fullerene is $20 \times 20 \times 20 \AA$. The cutoff energy in our calculation is $520 \mathrm{eV}$. All the calculation are relaxed to minimize the total energy of the system until a precision of $1 \mathrm{meV}$ was reached. Following the common way about the definition of adsorption energy [35], we calculate the adsorption energy per Li atom $\left(E_{a d}\right)$ as following

$E_{a d}=\frac{\left(E_{G+n L i}-E_{G}-n E_{L i}\right)}{n}$

where $E_{G+n L i}, E_{G}$ and $E_{L i}$ represent the energy of Li-carbon, nanostructured carbon and one lithium atom, respectively, $n$ is the number of $\mathrm{Li}$ atoms. The calculated adsorption energy include two parts: one is the interaction between Lithium ions and carbon atoms and the other comes from the interaction among $\mathrm{Li}$ ions. To understand the interaction between the $\mathrm{Li}$ and the graphene, we define the pure adsorption energy per Li atoms $\left(E_{\text {atom }}\right)$ as follows

$E_{\text {atom }}=\frac{\left(E_{G+n L i}-E_{G}-E_{n L i}\right)}{n}$

where $\left(E_{n L i}\right)$ represents the energy of all lithium atoms including the interaction themselves and $\left(E_{G}\right)$ represents the energy of deformed nanostructured carbon. It is noted that all adsorption energy obtained here is negative. For convenience, we use the absolute values in all following plots. In general, the larger adsorption energy of Li atoms on graphene implies higher lithium storage capacity.

\section{Results and discussion}

As mentioned above, we are interested in how grain boundary defects and curvature influence the binding between lithium ions and the carbon structures. Hence the binding energy of lithium adsorbed on different carbon structure is examined, including a single Li adsorbed on graphene with GB defects of different type, $\mathrm{Li}$ atoms adsorbed on carbon nanotubes, and binding between $\mathrm{Li}$ and fullerene.

\subsection{Li adsorbed on defective graphene}

We first show the adsorption energy for a single Li adsorbed on different position of pentagon-heptagon defects (Fig. 1a) in grain boundary of tilt angle $27.79^{\circ}[36]$ and a grain boundary (GB) composed of pentagon-octagon defects (Fig. 1b) and discrete vacancies. It is seen that the pure adsorption energy of Li atoms is effectively enhanced even in hexagonal rings near the defect (Table 1). The adsorption energy for Li in hexagonal rings decreases as they stay far away from the defective region, and finally approaches to a constant value of $-1.584 \mathrm{eV}$. The approximate range subjected to the influence of defect is about $7.48 \AA$. A structure full of pentagon-octagon defects are shown in Fig. 1c, where the binding energy is extremely high even if each ring binds to one atom. We also try to add Li atoms on the GB of different angles [36], as shown in Fig. 1d and e. It is seen that Li atoms prefer to adsorb on pentagon-heptagon defects. Comparing the pure adsorption energy of Li on GBs with different angles seen in Fig. 1f, there is a very slight difference. It is mainly when Li atoms are trapped in pentagonheptagon rings to give rise to the high binding energy between Li-C system.

We further calculate the density of states (DOS) of graphene 

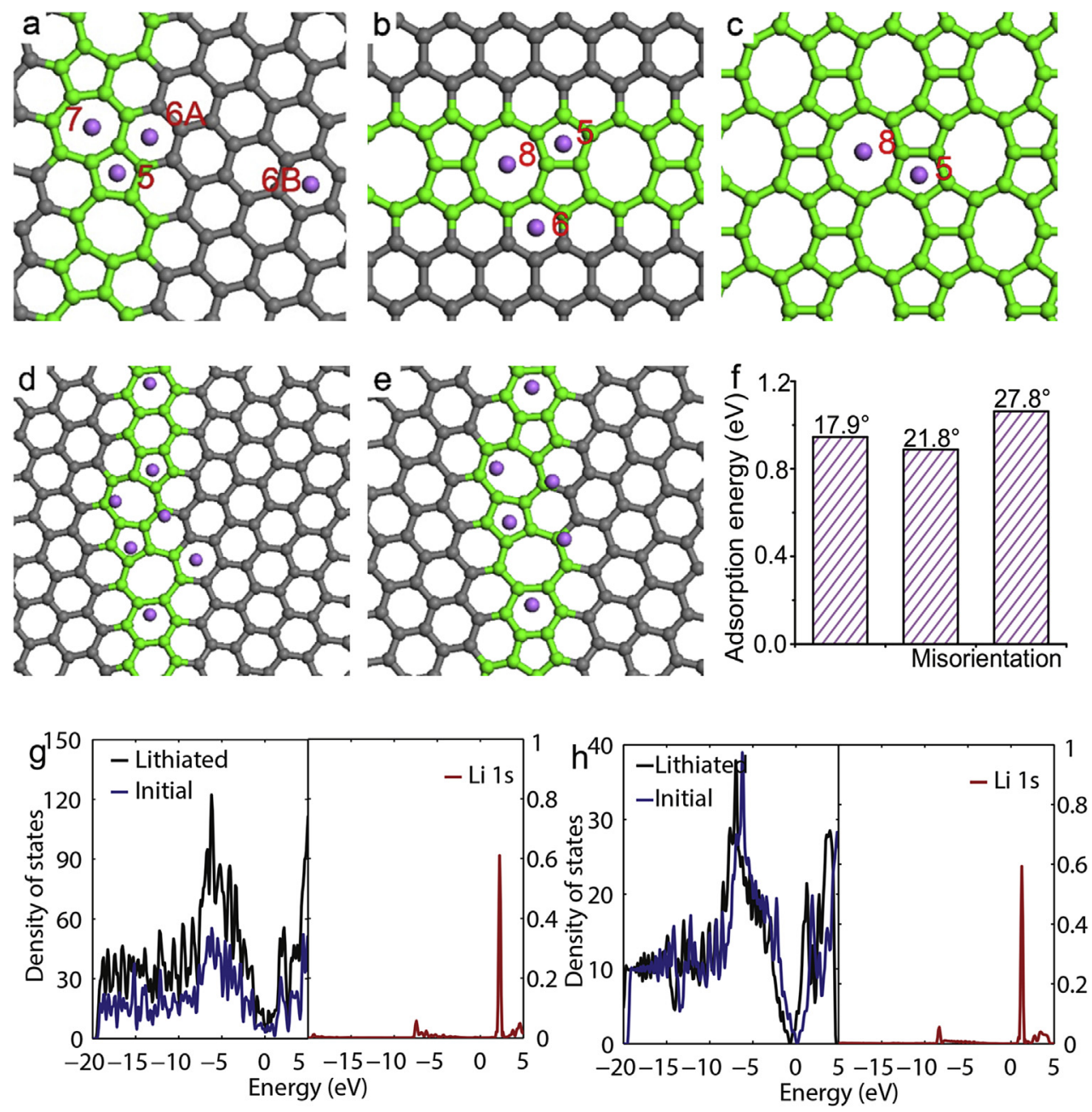

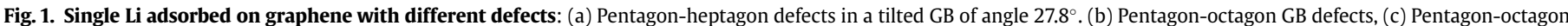

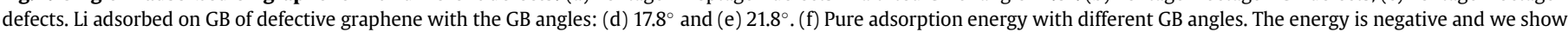

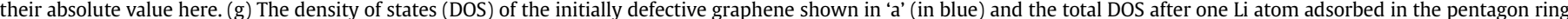

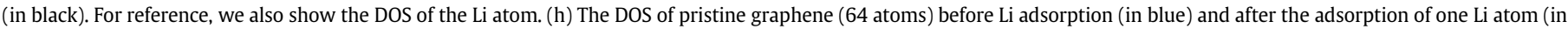
black). Again, the DOS of the Li atom. (A colour version of this figure can be viewed online.)

Table 1

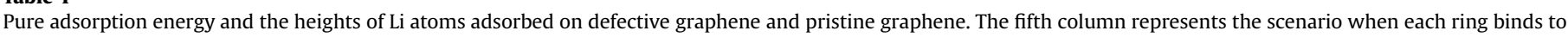
one Li atom.

\begin{tabular}{|c|c|c|c|c|}
\hline & Location & $E_{\text {atom }}(\mathrm{eV})$ (single Li) & Height $(\AA)$ & $E_{\text {atom }}(\mathrm{eV})$ (multiple Li) \\
\hline \multirow[t]{4}{*}{ 5-7 Defects (Fig. 1a) } & 5 & -2.338 & 1.797 & -0.150 \\
\hline & $6 \mathrm{~A}$ & -2.239 & 1.732 & \\
\hline & $6 \mathrm{~B}$ & -1.799 & 1.732 & \\
\hline & 7 & -2.248 & 1.600 & \\
\hline \multirow[t]{3}{*}{ 5-8 Defects (Fig. 1b) } & 5 & -2.635 & 1.785 & -0.222 \\
\hline & 6 & -2.332 & 1.736 & \\
\hline & 8 & -2.578 & 1.423 & \\
\hline \multirow[t]{2}{*}{ 5-8 Defects (Fig. 1c) } & 5 & -2.527 & 1.811 & -0.531 \\
\hline & 8 & -2.540 & 1.482 & \\
\hline Pristine & 6 & -1.584 & 1.724 & -0.031 \\
\hline
\end{tabular}

with grain boundary defects (Fig. 1g) and pristine graphene (Fig. 1h). As seen in the two figures, we see that pristine graphene is semiconductor, in good agreement with the previous report [15]. With lithium adsorption, the Fermi level of pristine graphene shifts to the conduction band. In contrast, defective graphene is in an electron-deficient state. Hence it prefers to attract electrons from $\mathrm{Li}$ atoms and owes larger adsorption energy.
To elucidate the mechanism influencing the adsorption energy for Li on defective graphene, we first examine the adsorption height of Li on defective graphene and pristine graphene. It is seen that the height of Li on pentagon defects is $0.073 \AA$ larger than that on pristine graphene, while that on heptagon defects is $0.124 \AA$ smaller than that on pristine graphene. The adsorption height on hexagon rings of defective graphene is similar as that on pristine graphene. It 
suggests that there is no direct correlation between the adsorption energy and height. We then compare the charge density as shown in Fig. 2. It is seen that the electron of $\mathrm{Li}$ is transferred to the surface of carbon in defective and pristine graphene, which forms ionic bonds. The Li atoms adsorbed on defective rings (pentagon ring, Fig. 2d and heptagon ring, Fig. 2f) transfer much more charge to $\mathrm{C}$ than the Li on pristine graphene (Fig. 2e), which is in consistent with the results based on the adsorption energy. We choose two cross-sections passing the pentagon-octagon defect in the grain boundary (Fig. $2 \mathrm{~g}$ ) to investigate its charge density transfer. For comparison, we also look at the charge density transfer in the pristine graphene (Fig. 2h). Fig. 2h shows that the transferred charge density on defective line is much higher than that on the pristine one. This trend holds for the defective graphene with pentagon-heptagon rings as well. The results suggest that the defect can influence the adsorption of Li on hexagon rings adjacent to it, while its influence on the hexagonal rings far way is mild. This is why the pure adsorption energy of Li on hexagonal rings near the defects is much greater than that far from defects.

We now consider the case that more $\mathrm{Li}$ atoms being adsorbed on defective graphene. In the case of each ring adsorbing one $\mathrm{Li}$, the ratio of $\mathrm{Li} / \mathrm{C}$ reaches $1: 2$. The adsorption energy is seen to decreases dramatically (Table 1). However, the defective graphene still has much higher adsorption energy than the pristine graphene. Furthermore, the adsorption energy of graphene with pure pentagon-octagon rings is up to $0.531 \mathrm{eV}$, approaching to that of $\mathrm{Li}$ on pristine graphene with $\mathrm{Li} / \mathrm{C}$ ratio of $1: 6(0.573 \mathrm{eV})$. This result proves that introduction of defective $5-7,5-8$ rings on graphene is feasible to realize high lithium storage in nanostructured carbon materials.

In contrast to GB defects composed of 5-7 rings and 5-8 rings, point vacancy may also present and influence of binding [25]. Three typical vacancies are shown in Fig. $3 a$ to $c$. The adsorption energy of Li to carbon in different region is given in Fig. 3d. Pure adsorption energy of Li on monovacancy defects is unexpectedly larger than that on pentagon-heptagon defects and pentagon-octagon defects. When two Li atoms adsorbed octagon ring in bivacancy defects, the pure adsorption energy per $\mathrm{Li}$ is up to $1.655 \mathrm{eV}$, which is much larger than that on pristine graphene $(0.543 \mathrm{eV})$. When three $\mathrm{Li}$ atoms adsorb on the enneagon ring in tetravacancy defects, the pure adsorption energy is still much larger than that on pristine graphene, although the repulsive interaction of Li atoms is intense. When more $\mathrm{Li}$ atoms are adsorpted, the adsorption energy is considerable higher than $\mathrm{Li}$ on pristine graphene. It hence suggests that even randomly bombed graphene with vacancies [37] could owe high lithium storage capacity.

We further investigate the adsorption property when more $\mathrm{Li}$ atoms being adsorbed on defective graphene. With two Li atoms adsorbed on the adjacent rings (Fig. 4a b c), we find the pure adsorption energy decreases compared with the case of single $\mathrm{Li}$ adsorption in several type of defects (Table 2 and Table 3). Further charge density results show that this reduction of adsorption
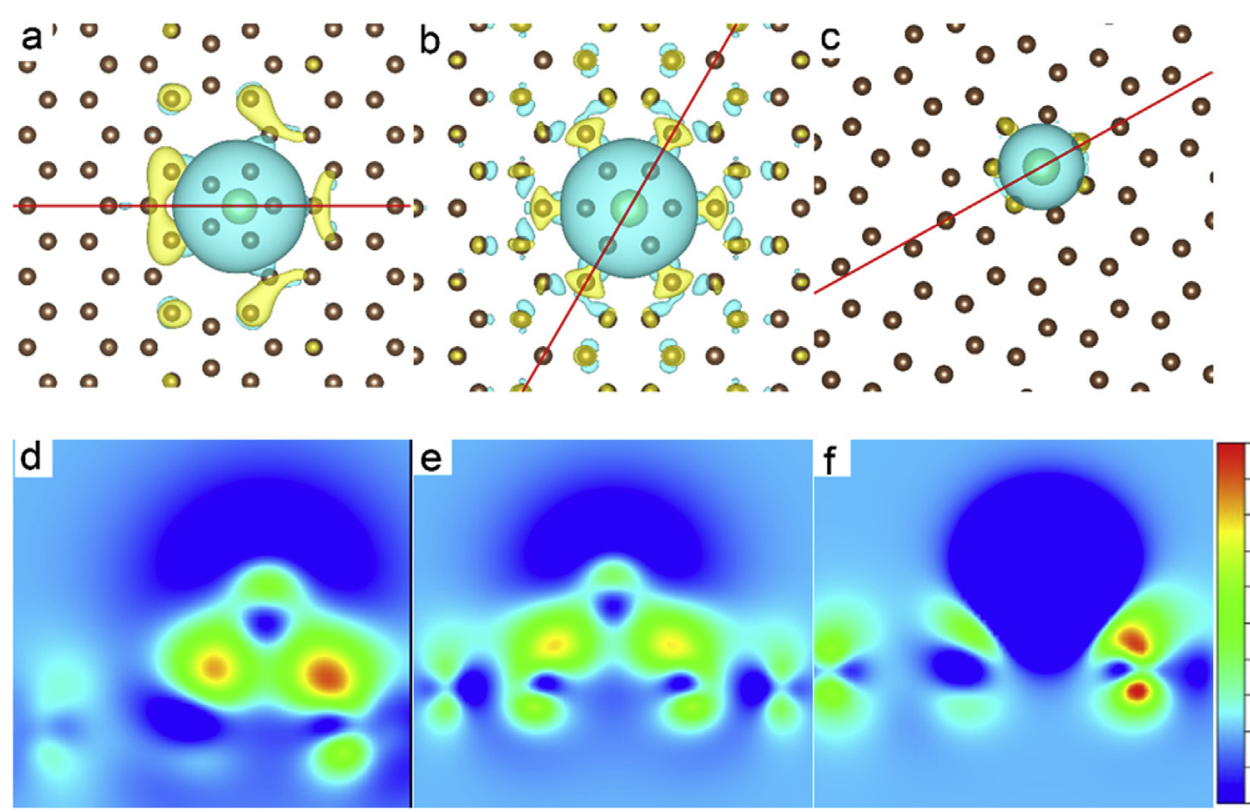

0.01
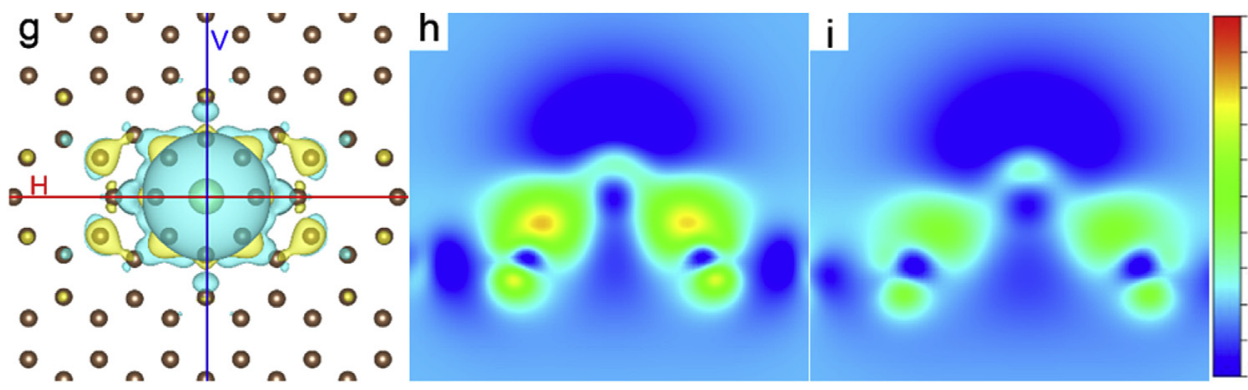

$-0.0025$

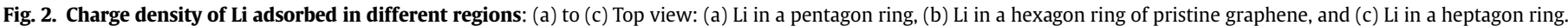

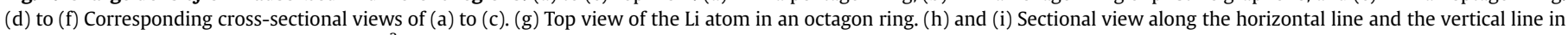
(g), respectively (Unit in the legend: e/bohr ${ }^{3}$ ). (A colour version of this figure can be viewed online.) 

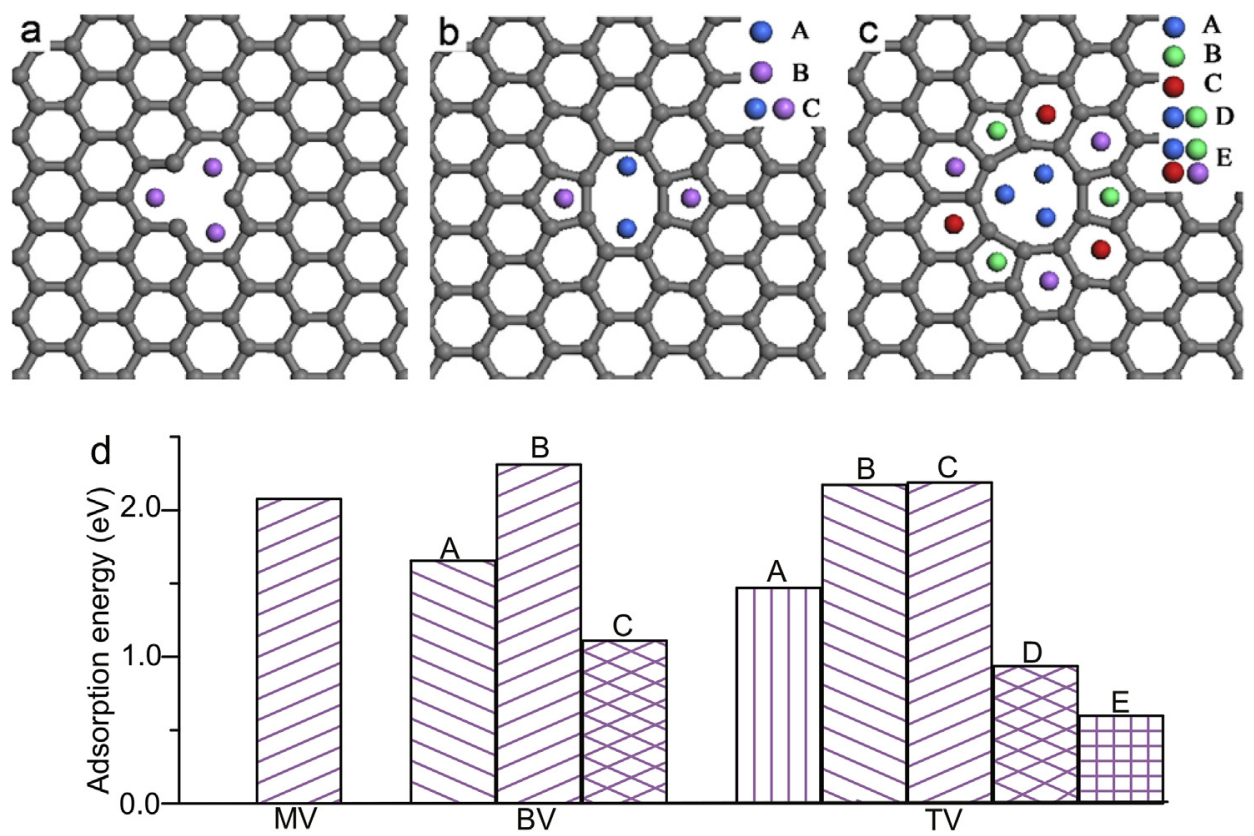

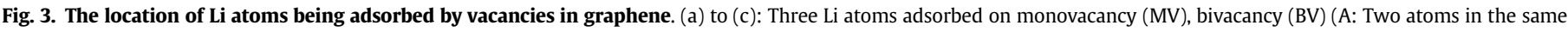

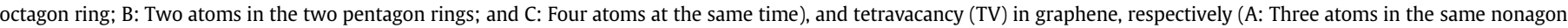

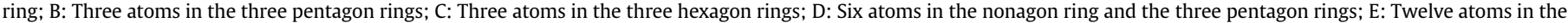

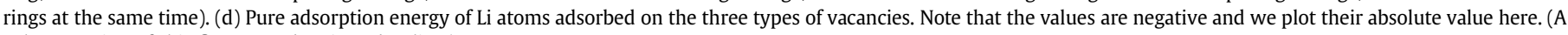
colour version of this figure can be viewed online.)

energy is not due to the ionic bond forming (Fig. 4d e f), but due to the repulsion between $\mathrm{Li}$ ions. Compare the adsorption of $\mathrm{Li}$ atoms on defective and pristine graphene, we find that pure adsorption energy of defective graphene is still much larger than that of pristine graphene. Although the Li atoms adsorbed on double pentagon rings repulse each other, the pure adsorption energy $(-1.709 \mathrm{eV})$ is still much higher than that on pristine graphene $(-0.838 \mathrm{eV})$. It suggests that defective graphene can absorb more Li atoms.

\subsection{Li atoms adsorbed on carbon nanotubes}

It has been shown that the decrease of pure adsorption energy of multiple $\mathrm{Li}$ atoms is due to the repulsion among $\mathrm{Li}$ atoms. One may postulate instantaneously that increasing the interval between Li atoms should enhance the pure adsorption energy. One feasible way is to adsorb Li atoms on curved graphene or carbon nanotubes. Indeed, our results in Fig. 5a show that with fixed C/Li ratio of 2:1,
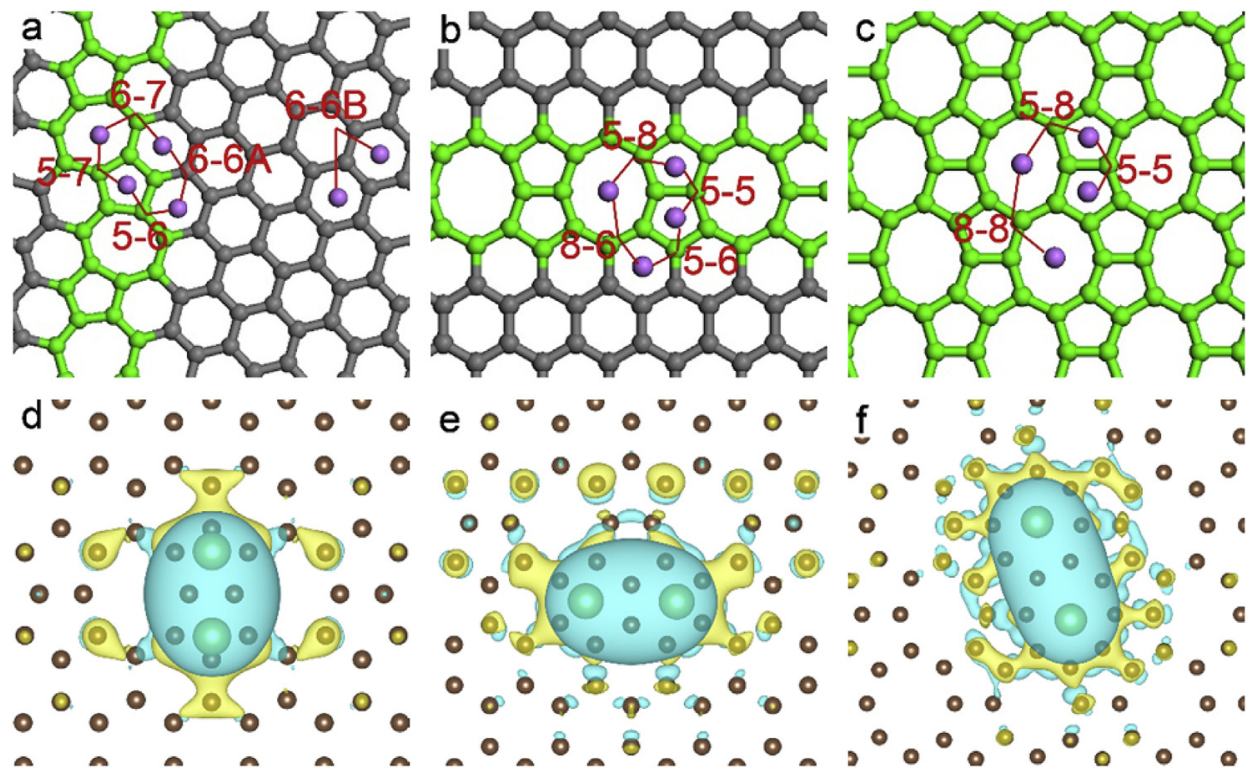

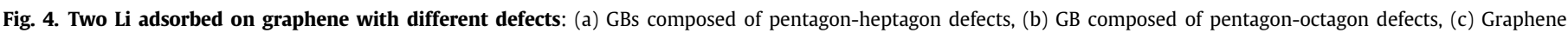

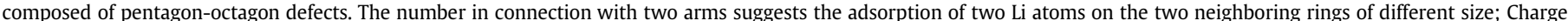

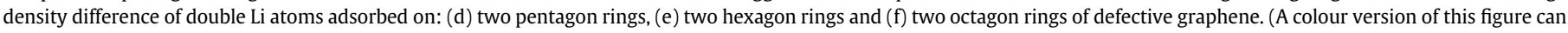
be viewed online.) 
Table 2

Pure adsorption energy of double Li atoms adsorbed on GB defects in graphene and pristine graphene.

\begin{tabular}{|c|c|c|c|c|c|c|}
\hline \multirow{3}{*}{$\begin{array}{l}\text { Location } \\
E_{\text {atom }}\end{array}$} & \multicolumn{5}{|c|}{ 5-7 defective graphene } & \multirow{2}{*}{$\frac{\text { Graphene }}{6-6}$} \\
\hline & $5-6$ & $5-7$ & $6-6 A$ & $6-6 B$ & $6-7$ & \\
\hline & -1.433 & -1.557 & -1.349 & -0.838 & -1.548 & -0.543 \\
\hline
\end{tabular}

Table 3

Pure adsorption energy and heights of double Li atoms adsorbed on pentagon-octagon defective graphene.

\begin{tabular}{|c|c|c|c|c|c|c|c|c|}
\hline \multirow{3}{*}{$\begin{array}{l}\text { Location } \\
E_{\text {atom }}\end{array}$} & \multicolumn{5}{|c|}{ 5-8 GB defects } & \multicolumn{3}{|c|}{ 5-8 defective graphene } \\
\hline & $5-5$ & $5-6$ & $5-8$ & $6-6$ & $8-6$ & $5-5$ & $5-8$ & $8-8$ \\
\hline & -1.709 & -1.572 & -1.829 & -1.439 & -1.799 & -1.612 & -1.726 & -2.010 \\
\hline
\end{tabular}

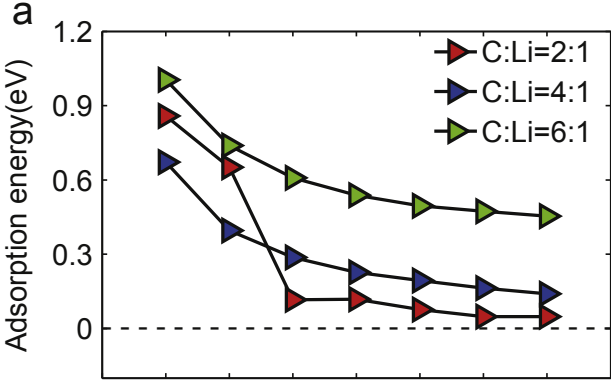

(33) (44) (55) (66) (77) (88) (99)
CNTs

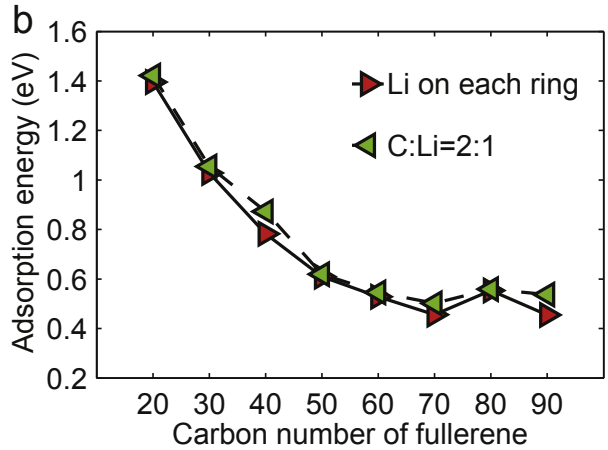

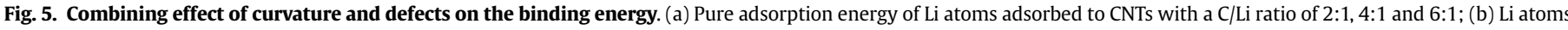

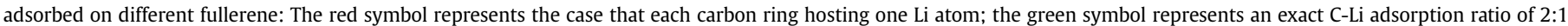
(absolute value). (A colour version of this figure can be viewed online.)

the pure adsorption energy of $\mathrm{Li}$ on $(3,3) \mathrm{CNT}$ is $-0.858 \mathrm{eV}$, which is greater than that with the C/Li ratio of either $4: 1$ or $6: 1$. For the two 'anomalous' points for $(3,3)$ and $(4,4)$ CNTS in Fig. 5a, they are resulted from the severely distorted deformation as the C/Li ratio of 2:1 is high. The trend becomes normal in CNTs of diameter greater than $\sim 1 \mathrm{~nm}$. When the curvature of CNTs decreases at every fixed C/ Li ratio, we find the pure adsorption energy also decreases. On the $(9,9)$ CNT with a C/Li ratio of 2:1, the pure adsorption energy approaches the value of $\mathrm{Li}$ on graphene $(-0.031 \mathrm{eV})$. The results from our calculations demonstrate that CNTs with large curvature have better lithium storage property.

\subsection{Li atoms adsorbed on fullerene}

We show in the previous sections how defects and curvature may influence the binding energy of Li on carbon. Now we explore the chance when both factors come into play. We conduct a series of calculations with $\mathrm{Li}$ atoms adsorbed on fullerenes of different radii. Each ring adsorbing one Li atom and adsorption with the ratio of Li/C about 1:2 are shown in Fig. 5b. Similar to the results for CNTs, the pure adsorption energy (with number of carbon atoms $\leq 50$ ) decreases with increasing radius. When the number of carbon atoms is more than 50, then pure adsorption energy tends to be stable. However, the value remains at a high level of $0.4 \mathrm{eV}$ for a fullerene of 90 atoms. This result suggests that both curvature and the pentagon defects play significant roles in enhancing Li atoms adsorption in fullerenes.

\section{Conclusions}

We have studied the Li atoms adsorbed on different kinds of defective graphene, and found the defect could enhance the $\mathrm{Li}$ adsorption by analyzing pure adsorption energy and charge density difference. We also investigated the Li atoms being adsorbed to armchair CNTs and found the adsorption energy increases with the curvature. Fullerene with smaller diameter favors lithium adsorption. Unlike CNTs, the adsorption energy of fullerene with larger diameter does not approach to the adsorption energy between $\mathrm{Li}$ and flat graphene. This suggests that the pentagon rings play a significant role to affect the adsorption. Our report suggests that both curvature and GB defects could enhance the adsorption of $\mathrm{Li}$ atoms on nanostructured carbon. The work supplies the theoretical basis for further improving the three key characteristics of lithium ion battery performance - capacity, voltage and energy density by making defective carbon structures and also by introducing rough morphology. While the larger adsorption energy of Li atoms on graphene normally leads to more storage capacity, it may also slow down the delithiation process as the energy barrier to separate $\mathrm{Li}$ atoms from graphene is higher. A later on investigation on the kinetics of $\mathrm{Li}-\mathrm{C}$ interaction is desired to illustrate such drawback.

\section{Acknowledgement}

The authors acknowledge support from the National Natural Science Foundation of China (NSFC) (Grant no. 11425211), and MOST 973 of China (Grant no. 2012CB937500). Fang thanks the support from NSFC (Grant no. 11572002).

\section{References}

[1] J.M. Tarascon, M. Armand, Issues and challenges facing rechargeable lithium batteries, Nature 414 (6861) (2001) 359-367.

[2] Y. Liu, et al., Mechanism of lithium insertion in hard carbons prepared by pyrolysis of epoxy resins, Carbon 34 (2) (1996) 193-200.

[3] E.J. Yoo, et al., Large reversible Li storage of graphene nanosheet families for 
use in rechargeable lithium ion batteries, Nano Lett. 8 (8) (2008) 2277-2282.

[4] V. Meunier, et al., Ab Initiolnvestigations of lithium diffusion in carbon nanotube systems, Phys. Rev. Lett. 88 (7) (2002).

[5] M. Khantha, et al., Interaction and concerted diffusion of lithium in a $(5,5)$ carbon nanotube, Phys. Rev. B 78 (11) (2008).

[6] L.A. Montoro, E.Y. Matsubara, J.M. Rosolen, Lithium intercalation into singlewalled carbon nanotubes network electrode: storage mechanisms and impurity effects, J. Power Sources 257 (2014) 205-212.

[7] H. Shimoda, et al., Lithium Intercalation into opened single-wall carbon nanotubes: storage capacity and electronic properties, Phys. Rev. Lett. 88 (1) (2001) 015502.

[8] B. Gao, et al., Enhanced saturation lithium composition in ball-milled singlewalled carbon nanotubes, Chem. Phys. Lett. 327 (1-2) (2000) 69-75.

[9] K.R. Kganyago, P.E. Ngoepe, Structural and electronic properties of lithium intercalated graphiteLiC6, Phys. Rev. B 68 (20) (2003).

[10] W. Koh, et al., Li adsorption on a graphene-fullerene nanobud system: density functional theory approach, RSC Adv. 5 (41) (2015) 32819-32825.

[11] W. Koh, et al., Mechanism of Li adsorption on carbon nanotube-fullerene hybrid system: a first-principles study, ACS Appl. Mater Interfaces 3 (4) (2011) 1186-1194.

[12] W. Koh, et al., Li adsorption on a fullerene-single wall carbon nanotube hybrid system: density functional theory approach, Curr. Appl. Phys. 14 (12) (2014) 1748-1754.

[13] W. Koh, et al., First-principles study of Li adsorption in a carbon nanotubefullerene hybrid system, Carbon 49 (1) (2011) 286-293.

[14] K. Chang, et al., Ultrathin MoS2/nitrogen-doped graphene nanosheets with highly reversible lithium storage, Adv. Energy Mater. 3 (7) (2013) 839-844.

[15] X. Wang, et al., First-principles study on the enhancement of lithium storage capacity in boron doped graphene, Appl. Phys. Lett. 95 (18) (2009) 183103.

[16] D.H. Wu, Y.F. Li, Z. Zhou, First-principles studies on doped graphene as anode materials in lithium-ion batteries, Theor. Chem. Accounts 130 (2-3) (2011) 209-213.

[17] Z. Zhou, et al., A first-principles study of lithium absorption in boron- or nitrogen-doped single-walled carbon nanotubes, Carbon 42 (12-13) (2004) 2677-2682.

[18] Y. Liu, et al., Feasibility of lithium storage on graphene and its derivatives, J. Phys. Chem. Lett. 4 (10) (2013) 1737-1742.

[19] Y.-X. Yu, Can all nitrogen-doped defects improve the performance of graphene anode materials for lithium-ion batteries? Phys. Chem. Chem. Phys. 15 (39) (2013) 16819-16827.
[20] J. Ye, et al., Universal roles of hydrogen in electrochemical performance of graphene: high rate capacity and atomistic origins, Sci. Rep. 5 (2015) 16190.

[21] J. Xiao, et al., Hierarchically porous graphene as a lithium-air battery electrode, Nano Lett. 11 (11) (2011) 5071-5078.

[22] L.-J. Zhou, Z.F. Hou, L.-M. Wu, First-principles study of lithium adsorption and diffusion on graphene with point defects, J. Phys. Chem. C 116 (41) (2012) $21780-21787$

[23] X. Fan, W.T. Zheng, J.-L. Kuo, Adsorption and diffusion of Li on pristine and defective graphene, ACS Appl. Mater. Interfaces 4 (5) (2012) 2432-2438.

[24] K. Nishidate, M. Hasegawa, Energetics of lithium ion adsorption on defective carbon nanotubes, Phys. Rev. B 71 (24) (2005).

[25] D. Datta, et al., Enhanced lithiation in defective graphene, Carbon 80 (2014) $305-310$.

[26] R. Mukherjee, et al., Photothermally reduced graphene as high-power anodes for lithium-ion batteries, Acs Nano 6 (9) (2012) 7867-7878.

[27] R. Mukherjee, et al., Defect-induced plating of lithium metal within porous graphene networks, Nat. Commun. (2014) 5.

[28] W.Y. Lee, S. Suzuki, M. Miyayama, Electrode properties of defect-introduced graphenes for lithium-ion batteries, in: Key Engineering Materials, Trans Tech Publ., 2014.

[29] X. Li, et al., Structurally tailored graphene nanosheets as lithium ion battery anodes: an insight to yield exceptionally high lithium storage performance, Nanoscale 5 (24) (2013) 12607-12615.

[30] G. Kresse, J. Furthmüller, Efficient iterative schemes for ab initio total-energy calculations using a plane-wave basis set, Phys. Rev. B 54 (16) (1996) 11169.

[31] G. Kresse, J. Hafner, Ab initio molecular dynamics for liquid metals, Phys. Rev. B 47 (1) (1993) 558.

[32] J.P. Perdew, K. Burke, M. Ernzerhof, Generalized gradient approximation made simple, Phys. Rev. Lett. 77 (18) (1996) 3865-3868.

[33] T.N.D. Alpha, et al., Structure of epitaxial graphene on $\operatorname{Ir}(111)$, New J. Phys. 10 (4) (2008) 043033.

[34] H.J. Monkhorst, J.D. Pack, Special points for Brillouin-zone integrations, Phys. Rev. B 13 (12) (1976) 5188-5192.

[35] C.B. Robledo, et al., First-principles studies of lithium storage in reduced graphite oxide, Electrochim. Acta 140 (2014) 232-237.

[36] Y. Wei, et al., The nature of strength enhancement and weakening by pentagon-heptagon defects in graphene, Nat. Mater 11 (9) (2012) 759-763.

[37] H. Wang, et al., Doping monolayer graphene with single atom substitutions, Nano Lett. 12 (1) (2012) 141-144. 